\title{
Dynamically stretched vortices as solutions of the 3D Navier-Stokes equations
}

\author{
J.D. Gibbon ${ }^{\mathrm{a}, *}$, A.S. Fokas ${ }^{\mathrm{a}}$, C.R. Doering ${ }^{\mathrm{b}}$ \\ a Department of Mathematics, Imperial College of Science, Technology and Medicine, London SW7 2BZ, UK \\ b Department of Mathematics, University of Michigan, Ann Arbor, MI 48109-1109, USA
}

Received 3 November 1998; accepted 19 March 1999

Communicated by A.C. Newell

\begin{abstract}
A well known limitation with stretched vortex solutions of the 3D Navier-Stokes (and Euler) equations, such as those of Burgers type, is that they possess uni-directional vorticity which is stretched by a strain field that is decoupled from them. It is shown here that these drawbacks can be partially circumvented by considering a class of velocity fields of the type $\boldsymbol{u}=\left(u_{1}(x, y, t), u_{2}(x, y, t), \gamma(x, y, t) z+W(x, y, t)\right)$ where $u_{1}, u_{2}, \gamma$ and $W$ are functions of $x, y$ and $t$ but not $z$. It turns out that the equations for the third component of vorticity $\omega_{3}$ and $W$ decouple. More specifically, solutions of Burgers type can be constructed by introducing a strain field into $\boldsymbol{u}$ such that $\boldsymbol{u}=(-(\gamma / 2) x-(\gamma / 2) y, \gamma z)+\left(-\psi_{y}, \psi_{x}, W\right)$. The strain rate, $\gamma(t)$, is solely a function of time and is related to the pressure via a Riccati equation $\dot{\gamma}+\gamma^{2}+p_{z z}(t)=0$. A constraint on $p_{z z}(t)$ is that it must be spatially uniform. The decoupling of $\omega_{3}$ and $W$ allows the equation for $\omega_{3}$ to be mapped to the usual general 2D problem through the use of Lundgren's transformation, while that for $W$ can be mapped to the equation of a $2 \mathrm{D}$ passive scalar. When $\omega_{3}$ stretches then $W$ compresses and vice versa. Various solutions for $W$ are discussed and some $2 \pi$-periodic $\theta$-dependent solutions for $W$ are presented which take the form of a convergent power series in a similarity variable. Hence the vorticity $\omega=\left(r^{-1} W_{\theta},-W_{r}, \omega_{3}\right)$ has nonzero components in the azimuthal and radial as well as the axial directions. For the Euler problem, the equation for $W$ can sustain a vortex sheet type of solution where jumps in $W$ occur when $\theta$ passes through multiples of $2 \pi$. (C1999 Elsevier Science B.V. All rights reserved.
\end{abstract}

Keywords: Stretched vortices; Burgers vortices; Lundgren's transformation; Vortex tubes; Passive scalar

\section{Introduction}

Stretched vortices of Burgers type, which are exact solutions of the Navier-Stokes equations, are often used as typical solutions to illustrate the tube-sheet paradigm of modern turbulence theory [1-9]. In reality they are pseudo3D in nature as they are composed of 2D flows superimposed on a 3D vorticity free strain field, a fact exploited by Lundgren in his transformation [2]. Depending on whether a tube or shear layer symmetry has been chosen,

\footnotetext{
${ }^{*}$ Corresponding author. E-mail: j.gibbon@ic.ac.uk.
} 
corresponding to uni-axial or bi-axial strain [3], they have the properties that the vorticity $\omega$ firstly lies respectively either along the axis of the tube or in the plane of the layer and secondly that it aligns with an eigenvector of the strain matrix $S$. While numerical simulations [10,11] and experiments [12] have shown that, in a spatially averaged sense, alignment of the vorticity vector $\omega$ with the intermediate eigenvector of $S$ is favoured (for a list of references see [13]), it is clear that local vorticity accumulation and alignment processes are more complicated than this $[14,15]$. For instance, the solution for the Burgers vortex has the drawback that it is stretched by a strain field that is decoupled from the flow around it and that its vorticity is unidirectional [1-3]. Complicated vortical structures caused by both vortex stretching and compression have a dynamic complexity that requires a more subtle theoretical explanation (see the recent review by Pullin and Saffman [16]). It is shown in this paper that it is possible to construct a more general class of stretched vortex solutions of the 3D Navier-Stokes (and Euler) equations which have nonzero components of vorticity in the radial, azimuthal and the axial directions and that these components are stretched or compressed by a strain that is dynamically coupled to the pressure field. Nevertheless, conventional Burgers vortices are generally the final asymptotic state if the strain rate $\gamma(t)$ is a positive function of time. $\gamma(t)$ itself plays a fundamental role in the coupling between the axial and horizontal components and that in turn, this object is driven by the second partial derivative of the pressure $p_{z z}$ through a Riccati equation.

These results are achieved in two stages. The first stage is to consider a generalisation of what is often referred to as 'columnar flow', the velocity field for which is given by

$$
\boldsymbol{u}=\left(u_{1}(x, y, t), u_{2}(x, y, t), \gamma(x, y, t) z+W(x, y, t)\right)
$$

with $u_{1}, u_{2}$ and $\gamma$ satisfying a continuity condition. The distinguished vertical variable $z$ appears only linearly in $u_{3}$, whereas $u_{1}, u_{2}, \gamma$ and $W$ are functions only of the horizontal co-ordinates and time. In polar co-ordinates the vorticity field takes the form

$$
\boldsymbol{\omega}=\left(\omega^{(r)}, \omega^{(\theta)}, \omega_{3}\right)=\left(r^{-1}\left(z \gamma_{\theta}+W_{\theta}\right),-\left(z \gamma_{r}+W_{r}\right), \omega_{3}\right)
$$

where the $r, \theta$ subscripts refer to partial derivatives. This shows that nonzero radial and azimuthal components are dependent on at least one of $\gamma$ or $W$ possessing some form of nontrivial spatial structure. In Section 2.1 it is shown that an unusual decoupling takes place between $\omega_{3}$ and $W$ with $\gamma$ satisfying a nonlinear equation which is related to the second $z$-derivative of the pressure $p_{z z}$ which is constrained to be spatially uniform. The second stage is to solve these equations for $\omega_{3}$ and $W$. To construct stretched vortex solutions of Burgers type it is necessary to be more specific with the velocity field in Eq. (1) and introduce a strain field structure into $u_{1}$ and $u_{2}$

$$
\boldsymbol{u}=\left(-\frac{1}{2} \gamma(t) x,-\frac{1}{2} \gamma(t) y, \gamma(t) z\right)+\left(-\psi_{y}, \psi_{x}, W\right)
$$

where $\omega_{3}=\psi_{x x}+\psi_{y y}$ with $\psi=\psi(x, y, t)$. Despite the fact that $\gamma$ has been chosen to be spatially uniform in Eq. (3), solutions for $W$ which are dependent on $r$ and $\theta$ can generate nonzero components $\omega^{(\theta)}$ and $\omega^{(r)}$. The unusual decoupling between $\omega_{3}$ and $W$ can be exploited advantageously by applying Lundgren's transformation which maps the equation for $\omega_{3}$ to a general $2 \mathrm{D}$ problem in the usual way [2]. In addition, the equation for $W$ is mapped to the equation for the evolution of a passive scalar following this flow. The role of $\gamma$ is particularly important in this process. One of the fundamental objects in Lundgren's transformation is the quantity $s(t)=\exp \int_{0}^{t} \gamma\left(t^{\prime}\right) \mathrm{d} t^{\prime}(\operatorname{see}$ Section 2.2), which obviously grows or decays depending on the sign of $\gamma$. When $s$ increases $\omega_{3}$ stretches, while $\omega^{(\theta)}$ and $\omega^{(r)}$ compress; $\omega$ therefore rotates to align more closely with the $z$-axis. Conversely, when $s$ decreases the opposite process occurs and $\omega$ rotates towards the horizontal plane. This effect is orchestrated by $p_{z z}$ through a Riccati equation for $\gamma$.

Perhaps the most spectacular solution of the Navier-Stokes equations is Lundgren's approximate 2D spiral solution for $\omega_{3}$. It is the only solution whose energy spectrum has a $k^{-5 / 3}$ factor [2]. In Section 3 it is shown how 
$2 \pi$-periodic $\theta$-dependent solutions for $W$ can be found in terms of a convergent power series solution in a similarity variable. The difference between this construction of $\theta$-dependent solutions for $W$, which are not obviously of spiral form, and that of Lundgren for $\omega_{3}$, is that the $u^{(\theta)}$ term in the Jacobian is taken from the exact point source solution for the Burgers vortex. Of course, $\theta$-dependence in $W$ results in a nonzero $\omega^{(r)}$ component.

In the case of the Euler equations it is shown in Section 4 that given suitable initial conditions, a vortex sheet-like solution can be found where a jump in $W$ occurs every time the azimuthal angle $\theta$ passes through $2 \pi$. This produces a $\delta$-function in the radial component of vorticity. In addition, the example of Stuart's vortices [17] is used to illustrate how a solution of the basic 2D equation can be used to construct a solution of the $W$ equation.

There exists a substantial body of literature on exact solutions of the Navier-Stokes equations of which the Burgers solutions are the most well known. In addition, there are also steady periodic ABC flows [18] and the Kelvin mode shear flow solutions of Craik and Criminale [19]. Takaoka has discussed a restricted case of the type of velocity field given in Eq. (3) in order to investigate reconnection processes but has $\psi_{x}$ and $\psi_{y}$ restricted to being functions of $t$ only [20]. With particular relevance to the class of flows given in Eq. (1) but with $W=0$, Sullivan [21] extended the steady solution for a viscous vortex embedded in a radially inward asymmetric stagnation point flow over a plane boundary [1,22]. This vortex naturally divides into two cells and the solution has been extended by Bellamy-Knights to take account of a moving separation surface between them [23].

\section{Decoupling in 3D Navier-Stokes solutions}

\subsection{The evolution of the columnar flow velocity field}

Consider the Navier-Stokes equations

$$
\frac{D \boldsymbol{u}}{D t}=v \Delta \boldsymbol{u}-\nabla p, \quad \operatorname{div} \boldsymbol{u}=0
$$

where the total derivative is given by

$$
\frac{D}{D t}=\frac{\partial}{\partial t}+\boldsymbol{u} \cdot \nabla
$$

$p=p(x, y, z, t)$ is the pressure and $\Delta$ is the full 3D Laplacian operator. Let $\boldsymbol{u}=\left(u_{1}, u_{2}, u_{3}\right)$ be a candidate velocity field solution of Eq. (4) taken in the form

$$
u_{1}=u_{1}(x, y, t), \quad u_{2}=u_{2}(x, y, t), \quad u_{3}=z \gamma(x, y, t)+W(x, y, t)
$$

with $z$ appearing only in $u_{3}$. With this velocity field the total derivative is now

$$
\frac{D}{D t}=\frac{\partial}{\partial t}+u_{1} \frac{\partial}{\partial x}+u_{2} \frac{\partial}{\partial y}+(z \gamma+W) \frac{\partial}{\partial z} .
$$

Note that whenever this operates on the variables $\omega_{3}, W$ and $\gamma$ (as well as $u_{1}$ and $u_{2}$ ), which are all functions of $x, y$ and $t$ but not $z$, then it behaves as a $2 \mathrm{D}$ operator. We also define the $2 \mathrm{D}$ version of the Laplacian operator as

$$
\Delta_{2}=\frac{\partial^{2}}{\partial x^{2}}+\frac{\partial^{2}}{\partial y^{2}}
$$

With $\boldsymbol{u}$ defined in Eq. (6) the vorticity vector in Cartesian co-ordinates for this flow is given by

$$
\boldsymbol{\omega}=\left(\omega_{1}, \omega_{2}, \omega_{3}\right)=\left(W_{y}+z \gamma_{y},-W_{x}-z \gamma_{x}, u_{2, x}-u_{1, y}\right) .
$$


where the $x, y$ subscripts refer to partial derivatives. This vorticity vector must satisfy

$$
\frac{D \omega}{D t}=S \omega+v \Delta \omega
$$

where $S$ is the strain matrix whose elements are

$$
S_{i j}=\frac{1}{2}\left(u_{i, j}+u_{j, i}\right) .
$$

The subtle nonlocal relations that hold between $u_{1}, u_{2}, W$ and the components of $\omega$ would not, at first glance, appear to engender optimism that any simple pattern could emerge. Remarkably, however, a decoupling occurs, the precise nature of which is expressed in the following.

Theorem 1. With the columnar type velocity field defined in Eq. (6), $\omega_{3}, W$ and $\gamma$ satisfy

$$
\begin{aligned}
& \frac{D \omega_{3}}{D t}=\gamma \omega_{3}+v \Delta_{2} \omega_{3}, \\
& \frac{D W}{D t}=-\gamma W+\nu \Delta_{2} W, \\
& \frac{D \gamma}{D t}+\gamma^{2}+p_{z z}(t)=v \Delta_{2} \gamma
\end{aligned}
$$

with $u_{1}$ and $u_{2}$ satisfying the continuity condition

$$
u_{1, x}+u_{2, y}+\gamma=0 .
$$

Remark 1. Note that the second partial $z$-derivative of the pressure $p_{z z}$ must be spatially uniform, a constraint necessary for the existence of such flows.

Remark 2. The calculations of this paper have been performed without the inclusion of rotation. Not surprisingly, the addition of this effect makes little difference as the proof will show; the inclusion of a $2 \boldsymbol{\Omega} \times \boldsymbol{u}$ in the Navier-Stokes equations adds no extra factor to the equation for $u_{3}$ if $\boldsymbol{\Omega}$ points in the $\hat{\boldsymbol{k}}$ direction. The theorem therefore remains, in essence, the same except for $\omega_{3} \rightarrow \omega_{3}+2 \Omega$, which corresponds to a change in the horizontal flow problem.

Proof. The evolution of the third velocity component $u_{3}=\gamma z+W$ in the Navier-Stokes equations is given by

$$
v\left(z \Delta_{2} \gamma+\Delta_{2} W\right)-p_{z}=\frac{D}{D t}(\gamma z+W)=z\left(\frac{D \gamma}{D t}+\gamma^{2}\right)+\left(\frac{D W}{D t}+\gamma W\right)
$$

which, on integration with respect to $z$, gives

$$
-p(x, y, z, t)=\frac{1}{2} z^{2}\left(\frac{D \gamma}{D t}+\gamma^{2}-v \Delta_{2} \gamma\right)+z\left(\frac{D W}{D t}+\gamma W-v \Delta_{2} W\right)+f(x, y, t) .
$$

However, from the first two components of the Navier-Stokes equations, we know that $p_{x}$ and $p_{y}$ must be independent of $z$. For this to be true the coefficients of $z$ and $z^{2}$ in Eq. (17) must necessarily satisfy

$$
\frac{D W}{D t}+\gamma W-v \Delta_{2} W=c_{1}(t)
$$

and

$$
\frac{D \gamma}{D t}+\gamma^{2}-v \Delta_{2} \gamma=c_{2}(t)
$$


$c_{1}(t)$ can be taken as zero without loss of generality as this term is like an acceleration of the co-ordinate frame in the $z$-direction. Equation (17) shows that $c_{2}(t)=-p_{z z}(t)$. This obviously restricts $p_{z z}$ to being spatially uniform. To find the evolution of $\omega_{3}$ we consider the strain matrix $S=\left\{S_{i j}\right\}$

$$
S=\left(\begin{array}{ccc}
u_{1, x} & (1 / 2)\left(u_{1, y}+u_{2, x}\right) & (1 / 2)\left(z \gamma_{x}+W_{x}\right) \\
(1 / 2)\left(u_{1, y}+u_{2, x}\right) & u_{2, y} & (1 / 2)\left(z \gamma_{y}+W_{y}\right) \\
(1 / 2)\left(z \gamma_{x}+W_{x}\right) & (1 / 2)\left(z \gamma_{y}+W_{y}\right) & \gamma
\end{array}\right) .
$$

Eqs. (9) and (20) show that

$$
(S \omega)_{3}=\gamma \omega_{3}
$$

so the third component of Eq. (10) shows that $\omega_{3}$ decouples from $W$ to give

$$
\frac{D \omega_{3}}{D t}=\gamma \omega_{3}+v \Delta_{2} \omega_{3} \text {. }
$$

Finally two checks should be applied. It is straightforward to demonstrate that the evolution of $\omega_{1}=z \gamma_{y}+W_{y}$ and $\omega_{2}=-\left(z \gamma_{x}+W_{x}\right)$ in Eq. (10) is consistent with Eqs. (12)-(15). It is also straightforward to demonstrate that the Poisson equation $-\Delta p=u_{i, j} u_{j, i}$ is also consistent with Eqs. (12)-(15).

\subsection{Results for a strain field}

It is instructive to be more specific and break $u_{1}$ and $u_{2}$ into a strain part and a $2 \mathrm{D}$ part in the conventional way

$$
u_{1}=-\frac{\gamma}{2} x-\psi_{y}, \quad u_{2}=-\frac{\gamma}{2} y+\psi_{x}
$$

by introducing a stream function $\psi(x, y, t)$. The variable $\gamma$, which now can be identified as the strain rate, is taken as a function of time only $\gamma=\gamma(t)$. The continuity condition is now satisfied. Equations (12) and (13) in Theorem 1 now become

$$
\begin{aligned}
& \frac{D \omega_{3}}{D t}=\gamma(t) \omega_{3}+v \Delta_{2} \omega_{3}, \\
& \frac{D W}{D t}=-\gamma(t) W+v \Delta_{2} W,
\end{aligned}
$$

where the total derivative is given by

$$
\frac{D}{D t}=\frac{\partial}{\partial t}-\frac{\gamma}{2}\left(x \frac{\partial}{\partial x}+y \frac{\partial}{\partial y}\right)+J_{x, y}(\psi, \cdot) .
$$

New co-ordinates can be found by Lundgren's transformation [2]

$$
\begin{aligned}
& s(t)=\exp \left(\int_{0}^{t} \gamma\left(t^{\prime}\right) \mathrm{d} t^{\prime}\right) \\
& \xi=s^{1 / 2} x, \quad \eta=s^{1 / 2} y, \quad \tau=\int_{0}^{t} s\left(t^{\prime}\right) \mathrm{d} t^{\prime} .
\end{aligned}
$$

The vorticity component $\omega_{3}$ and the velocity component $W$ can be rescaled into new variables thus

$$
\Omega_{3}(\xi, \eta, \tau)=s^{-1} \omega_{3}(x, y, t) \quad \text { and } \quad \mathcal{W}(\xi, \eta, \tau)=s W(x, y, t) .
$$


In the $(\xi, \eta)$ variables define the $2 \mathrm{D}$ Laplacian $\tilde{\Delta}_{2}$ as

$$
\tilde{\Delta}_{2}=\frac{\partial^{2}}{\partial \xi^{2}}+\frac{\partial^{2}}{\partial \eta^{2}}
$$

and the operator $D / D \tau$ as

$$
\frac{D}{D \tau}=\frac{\partial}{\partial \tau}+J_{\xi, \eta}(\Psi, \cdot)
$$

where $\Psi(\xi, \eta, \tau)=\psi(x, y, t)$. It is now easily shown that Eqs. (24) and (25), together with Eq. (26), can be transformed into the scaled variables $\Omega_{3}$ and $\mathcal{W}$ expressed in the new co-ordinate system $(\xi, \eta, \tau)$. The result can be summarised as follows:

Theorem 2. The quantity $\Omega_{3}(\xi, \eta, \tau)$ evolves according to the $2 D$ Navier-Stokes problem

$$
\frac{D \Omega_{3}}{D \tau}=v \tilde{\Delta}_{2} \Omega_{3}
$$

with $\Omega_{3}=\tilde{\Delta}_{2} \Psi$, whereas $\mathcal{W}(\xi, \eta, \tau)$ is a passive scalar and is found from solving the $2 D$ linear problem

$$
\frac{D \mathcal{W}}{D \tau}=\nu \tilde{\Delta}_{2} \mathcal{W}
$$

The strain rate $\gamma(t)$ is governed by the Riccati equation

$$
\dot{\gamma}+\gamma^{2}+p_{z z}(t)=0
$$

Remark 3. As Lundgren has shown [2], Eq. (32) is the standard 2D Navier-Stokes equation expressed in the $(\xi, \eta, \tau)$ variables. Solutions of this can then be mapped back into the original $3 D$ problem. $\mathcal{W}$ is a passive scalar driven by this flow and is solved from the linear Eq. (33) once derivatives of $\Psi$ have been determined from Eq. (32). In Sections 3 and 4 we show how this might be exploited through some examples.

Remark 4. When $\gamma<0$ it is possible that the domain $t \in[0, \infty]$ could map on to a finite section of the $\tau$-axis. For example, if $\gamma=-\gamma_{0}=$ constant with $\gamma_{0}>0$ then $s=\exp \left(-\gamma_{0} t\right)$ and $\tau=\gamma_{0}^{-1}\left[1-\exp \left(-\gamma_{0} t\right)\right]$. Hence $t \in[0, \infty]$ maps onto $\tau \in\left[0, \gamma_{0}^{-1}\right]$. If $\gamma$ is such that s decays less strongly then the $\tau$-axis could be infinite.

\subsection{A Riccati equation for $\gamma$}

Eq. (29) shows that the sign of $\gamma$ determines whether $\omega_{3}$ and $W$ grow or decay. The strain rate $\gamma$ satisfies an equation of Riccati type which can be linearized. Actually the linearizing transformation is none other than Eq. (27). Indeed $s$ satisfies

$$
\ddot{s}+\left\{p_{z z}(t)\right\} s=0 .
$$

Thus $s(t)$ is an eigenvector of the classical Sturm-Liouville equation corresponding to a zero eigenvalue. It is known (see for example [24]) that if $p_{z z}(t)$ remains positive for all $t$ then $s$ will develop a zero in finite time, in which case $\gamma \rightarrow \pm \infty$. We illustrate the relationship between $p_{z z}, s$ and $\gamma$ by considering some explicit examples. Let

$$
p_{z z}(t)=-a^{2}=\mathrm{const}
$$

then Eq. (35) yields

$$
s=\exp ( \pm a t) \quad \text { or } \quad s=\cosh a t
$$


Hence

$$
\gamma= \pm a \quad \text { or } \quad \gamma=a \tanh a t
$$

The second form of $\gamma$ above satisfies $\gamma \rightarrow a$ as $t \rightarrow \infty$. We note that a zero in $s$ will appear from the other hyperbolic solution where $\gamma$ blows up at $t=0$. Another form of $p_{z z}$ which also gives an explicit $\gamma$ is

$$
p_{z z}=a^{2}\left(2 \operatorname{sech}^{2} a t-1\right)
$$

which is positive at $t=0$ but becomes negative as $t$ increases. In this case $s=\operatorname{sech}$ at and so

$$
\gamma=-a \tanh a t
$$

Clearly $\gamma \rightarrow-a$ as $t \rightarrow \infty$. The case when $\gamma$ is spatially dependent is discussed in Section 5

It is worth noting that no other second spatial derivative other than $p_{z z}(t)$ drives $s(t)$ which, in turn, controls the growth or decay of the azimuthal and vertical vorticity components. The restriction that $p_{z z}$ must be spatially uniform does not apply to $p=p(x, y, z, t)$ itself as Eq. (17) shows that $p$ is a function of all three spatial variables with the horizontal part $f(x, y, t)$ being found by solving a 2D Poisson equation.

\section{Solutions of the Navier-Stokes equations}

In addition to the transformation that reduces the evolution of $\omega_{3}$ to a $2 \mathrm{D}$ problem, Lundgren also produced spiral solutions of the Euler and Navier-Stokes equations [2] (see also [16]). These solutions are for $\Omega_{3}$, and are therefore obtained from Eq. (32). Since this equation is decoupled from $\mathcal{W}$, the spiral solution is still appropriate here with $\mathcal{W}$ passively following this flow. Here we briefly discuss this solution in the context of this paper, to see if it is appropriate to use it when looking for exact solutions for $\mathcal{W}$. Indeed, how the Navier-Stokes spiral solution is constructed gives an idea of how $\theta$-dependent solutions for $\mathcal{W}$ might be found.

In the inviscid case, Lundgren's idea was to look for a solution of Eq. (32) for $\Omega_{3}$ in the form of a set of Dirac $\delta$-functions [2]

$$
\Omega_{3}(\rho, \theta, \tau)=2 \pi \sum_{j} \Gamma_{j}(\rho) \delta\left[\theta-\tilde{\Omega}(\rho) \tau-\theta_{j}\right]
$$

where $\rho$ is the 2D radial variable in the $\xi-\eta$ plane such that $\rho^{2}=\xi^{2}+\eta^{2}$. Equation (41) could be envisaged as representing a set of radial vortex sheets at angles $\theta_{j}$ and strengths $\Gamma_{j} . \tilde{\Omega}(\rho)=\rho^{-1} u^{(\theta)}$ is taken to be the average angular velocity. Clearly the sheets in Eq. (41) have a spiral structure of the form $\theta=\tilde{\Omega}(\rho) \tau$.

Viscous corrections to this for the Navier-Stokes equations were obtained by considering the full equation for $\Omega_{3}$

$$
\frac{\partial \Omega_{3}}{\partial \tau}+\tilde{\Omega}(\rho, \tau) \frac{\partial \Omega_{3}}{\partial \theta}=v\left(\frac{\partial^{2} \Omega_{3}}{\partial \rho^{2}}+\frac{1}{\rho} \frac{\partial \Omega_{3}}{\partial \rho}+\frac{1}{\rho^{2}} \frac{\partial^{2} \Omega_{3}}{\partial \theta^{2}}\right)
$$

with the $\theta$-dependence being $2 \pi$-periodic. The angular velocity $\tilde{\Omega}=\rho^{-1} \Psi_{\rho}$ was taken to be independent of $\tau$ on the grounds that it is a rapidly decaying function of $\tau$ and so can be replaced by its asymptotic value $\tilde{\Omega}(\rho)$. Lundgren then demonstrated that the viscous corrections to the Fourier series solution of Eq. (42) matched the Fourier series representation of Eq. (41) in the limit $v \rightarrow 0$.

In theory, this spiral solution for $\Omega_{3}$ could also be used for $\mathcal{W}$ but in practice the inviscid limit leads us into trouble in this case. It must be remembered that $\mathcal{W}$ is a velocity field and therefore the radial component of vorticity 
contains a $W_{\theta}$ term. In the inviscid limit this means we would have a derivative of a $\delta$-function, leading us into difficulties with generalised functions. To find simpler $\theta$-dependent solutions for $\mathcal{W}$ requires a modification of the idea used in Eq. (42) to which we turn in Section 3.2. Before this, however, we discuss axisymmetric solutions for $\Omega_{3}$ and $\mathcal{W}$.

\subsection{Axisymmetric solutions for $\mathcal{W}$}

Equation (32) implies that axisymmetric solutions for $\Omega_{3}$ can be found from the diffusion equation

$$
\frac{\partial \Omega_{3}}{\partial \tau}=v\left(\frac{\partial^{2} \Omega_{3}}{\partial \rho^{2}}+\frac{1}{\rho} \frac{\partial \Omega_{3}}{\partial \rho}\right) .
$$

A point source solution of this equation is

$$
\Omega_{3}(\rho, \tau)=\frac{a}{\tau} \exp \left[-\frac{\rho^{2}}{4 \nu \tau}\right] .
$$

This is the particular solution often used for the Burgers vortex where the parameter $a$ is any dimensionless constant. As an example let $\gamma=\gamma_{0}=$ constant where $\gamma_{0}>0$. Then $s=\exp \left(\gamma_{0} t\right)$ and $\tau=\gamma_{0}^{-1}\left(\exp \left(\gamma_{0} t\right)-1\right)$. Thus

$$
\omega_{3}=s \Omega_{3}=\frac{a \gamma_{0} \exp \left(\gamma_{0} t\right)}{\exp \left(\gamma_{0} t\right)-1} \exp \left\{-\frac{\gamma_{0} \exp \left(\gamma_{0} t\right) r^{2}}{4 v\left(\exp \left(\gamma_{0} t\right)-1\right)}\right\} .
$$

In the large time limit

$$
\lim _{t \rightarrow \infty} \omega_{3}=a \gamma_{0} \exp \left\{-\frac{\gamma_{0} r^{2}}{4 v}\right\} .
$$

For $\mathcal{W}$, the equivalent axisymmetric point source solution of Eq. (33) is

$$
\mathcal{W}(\rho, \tau)=\frac{\ell}{\tau} \exp \left\{-\frac{\rho^{2}}{4 v \tau}\right\}
$$

where, for dimensional reasons, we must take $a \equiv \ell$, a length, which could be any natural scale in the flow. The azimuthal vorticity component $\omega^{(\theta)}$ is given by

$$
\omega^{(\theta)}=-\frac{\partial W}{\partial r}=-s^{-1 / 2} \frac{\partial \mathcal{W}}{\partial \rho}
$$

so it follows that

$$
\frac{\omega^{(\theta)}}{r}=\frac{\ell}{2 \nu \tau^{2}} \exp \left[-\frac{\rho^{2}}{4 v \tau}\right] \text {. }
$$

If $\gamma=\gamma_{0}>0$ we conclude that whereas $\omega_{3}$ equilibrates to a finite constant value at any finite radius $r$, in contrast, $\omega^{(\theta)}$ decreases to zero.

Reversing the sign of $\gamma$ so that $\gamma=-\gamma_{0}=$ constant $\left(\gamma_{0}>0\right)$ we discover that $s=\exp \left(-\gamma_{0} t\right)$ and $\tau=$ $\gamma_{0}^{-1}\left(1-\exp \left(-\gamma_{0} t\right)\right)$. Hence $\tau \rightarrow \gamma_{0}^{-1}$ as $t \rightarrow \infty$ and $s \rightarrow 0$. In consequence

$$
\lim _{t \rightarrow \infty} \frac{\omega^{(\theta)}}{r}=\frac{\ell \gamma_{0}^{2}}{2 v} \text { and } \lim _{t \rightarrow \infty} \omega_{3}=0 .
$$

These two examples in which $\gamma$ takes opposite signs illustrates the point that $\omega_{3}$ and $\omega^{(\theta)}$ stretch or compress in opposite fashions. 


\subsection{Solutions for $\mathcal{W}$ dependent on $\theta$}

In looking for asymmetric solutions for $\mathcal{W}$ we use axisymmetric solutions for $\Omega_{3}$ (and hence $\Psi$ ) to find solutions for $\mathcal{W}$ that are dependent on $\theta$. It is not generally valid for a Navier-Stokes flow to admit solutions which jump each time $\theta$ passes through $2 \pi$ because of the problems arising from the $\mathcal{W}_{\theta \theta}$ term in the Laplacian. Solutions which are $2 \pi$-periodic in $\theta$ are more suitable. To achieve this goal consider solutions of Eq. (33) for $\mathcal{W}$ in terms of $\rho, \theta$

$$
\frac{\partial \mathcal{W}}{\partial \tau}+\frac{1}{\rho} J_{\rho, \theta}(\Psi, \mathcal{W})=v\left(\frac{\partial^{2} \mathcal{W}}{\partial \rho^{2}}+\frac{1}{\rho} \frac{\partial \mathcal{W}}{\partial \rho}+\frac{1}{\rho^{2}} \frac{\partial^{2} \mathcal{W}}{\partial \theta^{2}}\right)
$$

To calculate $\Psi_{\rho}$ in the Jacobian we integrate $\Omega_{3}=\tilde{\Delta}_{2} \Psi$ to obtain

$$
\frac{1}{\rho} \frac{\partial \Psi}{\partial \rho}=\frac{1}{\rho^{2}} \int_{0}^{\rho} \Omega_{3}\left(\rho^{\prime}, \tau\right) \rho^{\prime} \mathrm{d} \rho^{\prime} .
$$

and use the point source solution (44) to obtain

$$
\frac{1}{\rho} \frac{\partial \Psi}{\partial \rho}=v \frac{f(\zeta)}{\rho^{2}}
$$

where

$$
\zeta=\frac{\rho^{2}}{v \tau}
$$

and

$$
f(\zeta)=2 a\left\{1-\exp \left(-\frac{\zeta}{4}\right)\right\}
$$

This procedure is similar to that used in Eq. (42) except that we are using the point source solution instead of the $\tilde{\Omega}(\rho)$ factor used by Lundgren. Hence we have an inbuilt $\tau$-dependence in the coefficient $u^{(\theta)}$. The partial differential equation (51) in three variables $(\rho, \tau, \theta)$ can now be transformed into one in two variables $(\zeta, \theta)$

$$
\frac{\partial^{2} \mathcal{W}}{\partial \zeta^{2}}+\left(\frac{1}{4}+\frac{1}{\zeta}\right) \frac{\partial \mathcal{W}}{\partial \zeta}+\frac{1}{4 \zeta^{2}}\left(\frac{\partial^{2} \mathcal{W}}{\partial \theta^{2}}-f(\zeta) \frac{\partial \mathcal{W}}{\partial \theta}\right)=0
$$

In order to find separable solutions of this it is convenient to consider the complex function $\mathcal{V}$ related to $\mathcal{W}$ by

$$
\mathcal{W}=\operatorname{Re}\{\mathcal{V}\}
$$

which satisfies

$$
\frac{\partial^{2} \mathcal{V}}{\partial \zeta^{2}}+\left(\frac{1}{4}+\frac{1}{\zeta}\right) \frac{\partial \mathcal{V}}{\partial \zeta}+\frac{1}{4 \zeta^{2}}\left(\frac{\partial^{2} \mathcal{V}}{\partial \theta^{2}}-f(\zeta) \frac{\partial \mathcal{V}}{\partial \theta}\right)=0
$$

Now we look for separable solutions which are $2 \pi$-periodic in $\theta$

$$
\mathcal{V}=\mathcal{U}(\zeta) \mathrm{e}^{\mathrm{i} n \theta}
$$

where $n$ is an integer. The complex function $\mathcal{U}(\zeta)$ satisfies

$$
\frac{\mathrm{d}^{2} \mathcal{U}}{\mathrm{d} \zeta^{2}}+\left(\frac{1}{4}+\frac{1}{\zeta}\right) \frac{\mathrm{d} \mathcal{U}}{\mathrm{d} \zeta}-\left(\frac{n^{2}+\mathrm{i} n f(\zeta)}{4 \zeta^{2}}\right) \mathcal{U}=0
$$


where $f(\zeta)$ is defined in Eq. (55). This ordinary differential equation has a regular singular point at $\zeta=0$ and an irregular singular point at infinity. Neglecting the exponential part of $f(\zeta)$ when $|\zeta|$ is large, the appropriate asymptotic expansion in the latter case is $[25,26]$

$$
\mathcal{U}_{n}=\mathrm{e}^{-\zeta / 4} \sum_{p=1}^{\infty} \frac{b_{p}}{\zeta^{p}}
$$

In the finite $\zeta$-plane, $\mathcal{U}$ possesses a convergent power series solution whose form is dictated by its associated indicial equation. For small $\zeta, f(\zeta)$ takes the form $f(\zeta) \sim a \zeta / 2$ so the two roots of the indicial equation, $\pm(1 / 2) n$, differ by an integer. Thus one of the two solutions has a logarithmic singularity at $\zeta=0$. Excluding this solution, it follows that the physically admissible solution of Eq. (60) is given by the series

$$
\mathcal{U}_{n}=\zeta^{n / 2} \sum_{m=0}^{\infty} a_{m} \zeta^{m}, \quad n \neq 0
$$

which converges for all finite $\zeta$. Therefore our admissible solution for $\mathcal{W}$ is

$$
\mathcal{W}=\operatorname{Re} \sum_{n \neq 0} \mathcal{U}_{n} \mathrm{e}^{\mathrm{i} n \theta}
$$

\section{Results for the Euler equations}

For the flow with the velocity field given in Eq. (1), and in the case of zero viscosity, an immediate corollary of Theorem 1 is

$$
\frac{D\left(\omega_{3} W\right)}{D t}=0
$$

This result is independent of $\gamma$ and demonstrates in the simplest manner possible how $\omega_{3}$ and $W$ must increase and decrease in an opposite fashion. To see how $\omega_{3}$ and $W$ evolve individually we must restrict the flow to that of the strain field of Eq. (23). In this case, Eqs. (32) and (33) in Theorem 1 reduce to

$$
\frac{D \Omega_{3}}{D \tau}=\frac{\partial \Omega_{3}}{\partial \tau}+J_{\xi, \eta}\left(\Psi, \Omega_{3}\right)=0
$$

and

$$
\frac{D \mathcal{W}}{D \tau}=\frac{\partial \mathcal{W}}{\partial \tau}+J_{\xi, \eta}(\Psi, \mathcal{W})=0
$$

where $\Omega_{3}=\Psi_{\xi \xi}+\Psi_{\eta \eta}$. Eq. (65) is no more than the standard 2D Euler problem in Lundgren's variables. Equation (66) for $\mathcal{W}$ is the equation of a 2D passive scalar following this flow. Many solutions of the 2D Euler equations are catalogued in Saffman's book [4] and also in [27-31]. Hence we look here at only two examples. In the first example we consider Eq. (66) in $(r, \theta, t)$ co-ordinates to look at the evolution of a passive vortex sheet. In the second we consider the case of Stuart's vortices using $(\xi, \eta, \tau)$ variables.

\subsection{Solutions for $\mathcal{W}$ representing a vortex sheet}

Consider Eq. (66) for $\mathcal{W}$ written in terms of $r, \theta, t$ and $\psi$

$$
\frac{\partial \mathcal{W}}{\partial t}-\left(\frac{\gamma r}{2}\right) \frac{\partial \mathcal{W}}{\partial r}+\frac{1}{r} J_{r, \theta}(\psi, \mathcal{W})=0
$$


We take a similar approach to that of the previous section for the Navier-Stokes equations where axisymmetric solutions for $\omega_{3}$ and $\psi$ from Eq. (65) were used to look for asymmetric solutions for $\mathcal{W}$. Eq. (67) is a linear first order equation with variable coefficients

$$
\frac{\partial \mathcal{W}}{\partial t}-\left(\frac{\gamma r}{2}\right) \frac{\partial \mathcal{W}}{\partial r}+\left(\frac{1}{r} \frac{\partial \psi}{\partial r}\right) \frac{\partial \mathcal{W}}{\partial \theta}=0
$$

No discontinuities occur if initial data is $2 \pi$-periodic but if it is not then $\mathcal{W}$ must undergo a jump every time $\theta$ passes through $2 \pi$. Hence $\omega^{(r)}=r^{-1} \partial W / \partial \theta$ must have a $\delta(\theta)$-function structure representing a vortex sheet. In terms of characteristics in the $(r, \theta)$ plane, rays will satisfy

$$
\frac{\mathrm{d} r}{\mathrm{~d} t}=-\frac{\gamma r}{2}, \quad \frac{\mathrm{d} \theta}{\mathrm{d} t}=\frac{1}{r} \frac{\partial \psi}{\partial r} .
$$

On these rays $r=s^{-1 / 2}$. Thus when $s$ is increasing the sheet rolls up whereas when $s$ is decreasing the sheet rolls out and the evolution of $\theta(t)$ on these rays is driven by $\psi_{r}$. The whole structure is passive as it obviously advects with the 2D part of the flow. One physical analogy is that the sheet is akin to a flag, attached to a flagpole, the latter representing the tubular structure in $\omega_{3}$. It is worth noting that as well having a $\delta(\theta)$-function structure in $\omega^{(r)}$ representing a vortex sheet, across which the pressure is continuous, we also have a jump in $\theta$ in $\omega^{(\theta)}=-\partial W / \partial r$.

\subsection{Stuart's vortices}

The steady 'cat's eye' type solution (static in the sense of being independent of $\tau$ ) has been discussed by Stuart [17] who took $\Omega_{3}$ as a function of $\Psi$ only. This choice makes the Jacobian nonlinearity in Eq. (65) identically zero. Specifically he took

$$
\Omega_{3}=\Psi_{\xi \xi}+\Psi_{\eta \eta}=\mathrm{e}^{-2 \Psi} .
$$

The choice of the exponential on the right hand side makes Eq. (70) into Liouville's equation which can be solved exactly

$$
\Psi=\log \left(C \cosh \eta+\sqrt{C^{2}-1} \cos \xi\right) .
$$

When $1 \leq C \leq \infty$ the stream lines have a cat's eye structure. In the limit $C \rightarrow 1$ this gives the 2D velocity component $\partial \Psi / \partial \eta=\tanh \eta$ which corresponds to a homogeneous shear layer profile. The limit $C \rightarrow \infty$ gives the stream function for a $2 \mathrm{D}$ array of point vortices. For the $\mathcal{W}$ equation we know that $\mathcal{W}$ is a passive scalar following this flow; because of this we have the freedom to choose $\mathcal{W}$ also as an arbitrary function of $\Psi$. A convenient choice is $\mathcal{W}=\Omega_{3}=\mathrm{e}^{-2 \Psi}$ which means that

$$
\mathcal{W}=\left(C \cosh \eta+\sqrt{C^{2}-1} \cos \xi\right)^{-2} .
$$

This solution has a $\mathcal{W}=\operatorname{sech}^{2} \eta$ profile in the $C=1$ limit. The two components of vorticity $\omega_{1}$ and $\omega_{2}$ now become

$$
\omega_{1}=s^{-1 / 2} \Omega_{1}=\frac{-2 s^{-1 / 2} C \sinh \eta}{\left(C \cosh \eta+\sqrt{C^{2}-1} \cos \xi\right)^{3}}
$$

and

$$
\omega_{2}=s^{-1 / 2} \Omega_{2}=\frac{-2 s^{-1 / 2} \sqrt{C^{2}-1} \sin \xi}{\left(C \cosh \eta+\sqrt{C^{2}-1} \cos \xi\right)^{3}} .
$$


Not surprisingly these two components become significant when $s$ is decreasing. While they are independent of the 'time' variable $\tau$ they are not steady in real time $t$ which is built into $\xi$ and $\eta$ through $s(t)$.

\section{Conclusion}

The conclusion reached in this paper is that it is possible to extend the Burgers vortex to a more general class of vortices which have horizontal components of vorticity which interact with the axial component in an oppositional manner. While we have produced analytical solutions for $W$ in Section 3 in order to illustrate the stretching and compressive processes involved, it is obvious that because $\mathcal{W}$ is a passive scalar, a class of numerical solutions could be found which would depend on the underlying 2D flow problem for $\Omega_{3}$ and on the initial conditions for $\mathcal{W}$. The stretching and compressive processes in the problem are driven by $\gamma$ which, in turn, is controlled by $p_{z z}(t)$. Apparently this is an arbitrary function but we interpret its physical role as simply being the way the vortex connects with, and is influenced by, the rest of the flow. Having to keep $\gamma$ uniform in space unfortunately defeats the object of trying to establish a connection between the strain and the vorticity field. This would require $\gamma$ to have some form of spatial structure. Hence we replace Eq. (23) with

$$
\boldsymbol{u}=(-\beta x,-\beta y, \gamma z)+\left(-\psi_{y}, \psi_{x}, W\right),
$$

where $\beta=\beta(r, \theta, t)$ and $\gamma=\gamma(r, \theta, t)$. Theorem 1 still holds but the continuity condition needs to be recomputed to give

$$
\gamma=2 \beta+r \frac{\partial \beta}{\partial r}
$$

whence $\beta$ becomes

$$
\beta=\frac{1}{r^{2}} \int_{0}^{r} r^{\prime} \gamma\left(r^{\prime}, \theta, t\right) \mathrm{d} r^{\prime}+\frac{\Gamma(\theta, t)}{r^{2}} .
$$

One must however revert to using the total derivative in Eq. (14) so the equation for $\gamma$ is now

$$
\frac{\partial \gamma}{\partial t}-\beta r \frac{\partial \gamma}{\partial r}+\frac{1}{r} J_{r, \theta}(\psi, \gamma)+\gamma^{2}+p_{z z}(t)=v \Delta_{2} \gamma .
$$

Eqs. (77) and (78) are a pair of integro-partial differential equations in $r, \theta$ and $t$ driven by $p_{z z}(t)$. It is an open question whether this equation has any spatially dependent solutions for $\gamma$, even axisymmetric ones, when $p_{z z}(t)$ is uniform in the spatial variables. The possibility of using Lundgren's transformation is also sacrificed because $\gamma$ and $s$ are space dependent.

The spatial uniformity of $p_{z z}(t)$ required by Theorem 1 is a dramatic constraint. In Navier-Stokes turbulence, vortices are observed to have finite lifetimes and the mechanisms proposed in this paper may have a bearing on this. The ideal flows discussed here are infinite in domain and energy but, in reality, one could expect flows of this type to have $p_{z z}$ spatially uniform only over a finite region. It would be interesting to investigate what global conditions might be needed to achieve this. If the spatial uniformity constraint on $p_{z z}(t)$ failed in a given region then a vortex tube could break down and its vorticity concentration dissolve. Moreover, when $\gamma$ is uniform in space the vorticity solutions displayed in this paper are uniform in the variable $z$ and are therefore tube-like in character with a uniformity in $z$. Helical structures in 3D flows [32] are much more difficult to find and require a more subtle $z$-structure in the velocity and vorticity field than that used in Eq. (1). For the latter, if asymmetric solutions are taken for $W$ as in Section 3 then these tubes will possess a rich internal radial and azimuthal structure. The inclusion of the component $W$, however, means that $\omega$ typically lies neither in the horizontal plane nor along the $z$-axis, nor will it 
align with an eigenvector of $S$ as it does for the standard Burgers solutions. Let us illustrate this with two examples. Consider the stretching rate $\alpha$ which is defined in terms of the strain matrix $S$. This object has been discussed in some detail in [13] and can be expressed as a Biot-Savart type of integral [33,34]

$$
\alpha=\frac{\omega \cdot S \omega}{\omega \cdot \omega} .
$$

Consider first $\gamma=\gamma_{0}=$ constant where $\gamma_{0}>0$. We know in this case that $s$ is increasing exponentially so $\omega_{3}$ grows but $W$ decays. In the large time limit $\omega$ will rotate to the vertical position and $\alpha \rightarrow \gamma_{0}$. Consider now the second case when $\gamma=-\gamma_{0}=$ constant with $\gamma_{0}>0$. $s$ decreases exponentially so $\omega_{3}$ decays but $W$ grows. In the large time limit $\omega$ will move into the horizontal plane and $\alpha \rightarrow \gamma_{0} / 2$. The two examples discussed above are ideal cases in which $\gamma$ remains constant in magnitude and sign and in both cases $\omega$ will asymptotically align with an eigenvector of $S$. In a real flow, however, $\gamma$ will vary with time and therefore $\omega$ will rotate between the $z$-axis and the horizontal plane, thereby moving out of alignment with the eigenvectors of $S$. In a given region, however, if the strain is uniaxial for large times then $\gamma$ ends up as a positive function. In this case, the axial component of vorticity wins out and the conventional Burgers vortex is the final asymptotic state.

\section{Acknowledgements}

We are thankful to Darren Crowdy, Rupert Ford, John Gibbons, Darryl Holm, Bob Kerr, Robert Krasny, Frank Leppington, Derek Moore, Koji Ohkitani, Trevor Stuart and Arkady Tsinober for helpful conversations.

\section{References}

[1] J.M. Burgers, A mathematical model illustrating the theory of turbulence, Adv. Appl. Math. 1 (1948) 171.

[2] T. Lundgren, Strained spiral vortex model for turbulent fine structure, Phys. Fluids 25 (1982) 2193.

[3] H.K. Moffatt, S. Kida, K. Ohkitani, Stretched vortices - the sinews of turbulence; large-Reynolds-number asymptotics, J. Fluid Mech. 259 (1994) 241.

[4] P.G. Saffman, Vortex Dynamics, Cambridge University Press, Cambridge, 1993.

[5] U. Frisch, Turbulence: The Legacy of A.N. Kolmogorov, Cambridge University Press, Cambridge, 1995.

[6] A. Majda, Vorticity and the mathematical theory of incompressible fluid flow, Comm. Pure and Appl. Math. 39 (1986) 187.

[7] A. Majda, Vorticity, turbulence and acoustics in fluid flow, SIAM Rev. 33 (1991) 349.

[8] T. Kambe, A class of exact solutions of the Navier-Stokes equations, Fluid Dyn. Res. 1 (1986) 21.

[9] G. He, S. Chen, R.H. Kraichnan, R. Zhang, Y. Zhou, Statistics of dissipation and enstrophy induced by localised Burgers vortices, Phys. Rev. Lett. 81 (1998) 4636.

[10] W. Ashurst, A. Kerstein, R. Kerr, C. Gibson, Alignment of vorticity and scalar gradient with strain rate in simulated Navier-Stokes turbulence, Phys. Fluids 30 (1987) 2343.

[11] A. Vincent, M. Meneguzzi, The dynamics of vorticity tubes of homogeneous turbulence, J. Fluid Mech. 258 (1994) 245.

[12] A. Tsinober, E. Kit, T. Dracos, Experimental investigation of the field of velocity gradients in turbulent flows, J. Fluid Mech. 242 (1992) 169.

[13] B. Galanti, J.D. Gibbon, M. Heritage, Vorticity alignment results for the 3D Euler and Navier-Stokes equations, Nonlinearity 10 (1997) 1675.

[14] A. Tsinober, L. Shtilman, H. Vaisburd, A study of properties of vortex stretching and enstrophy generation in numerical and laboratory turbulence, Fluid Dyn. Res. 21 (1997) 477.

[15] A. Tsinober, Dynamics and statistics of concentrated vortices in turbulent flows, in: EUROMECH lecture, Colloquium 364, Marseille, France, 1997.

[16] D.I. Pullin, P.G. Saffman, Vortex dynamics, Ann. Rev. Fluid Mech. 30 (1998) 31.

[17] J.T. Stuart, On finite amplitude oscillations in laminar mixing layers, J. Fluid Mech. 29 (1967) 417.

[18] S. Childress, New solutions of the kinematic dynamo problem, J. Math. Phys. 11 (1970) 3063.

[19] A.D. Craik, W.O. Criminale, Evolution of wave-like disturbances in shear flows: a class of exact solutions of the Navier-Stokes equations, Proc. R. Soc. London, Ser. A 406 (1986) 13. 
[20] M. Takaoka, Straining effects and vortex reconnection of solutions to the 3-D Navier-Stokes equations, J. Phys. Soc. Jpn 60 (1991) 2602.

[21] R.D. Sullivan, A two-cell solution of the Navier-Stokes equations, J. Aero/Space Sci. 26 (1959) 26.

[22] N. Rott, On the viscous core of a vortex line II, Z.A.M.P. 10 (1959) 73.

[23] P.G. Bellamy-Knights, An unsteady two-cell vortex solution of the Navier-Stokes equations, J. Fluid Mech. 41 (1970) 673.

[24] E. Hille, Lectures on Ordinary Differential Equations, Addison-Wesley, London, 1969.

[25] E.L. Ince, Ordinary Differential Equations, Dover Publications, New York, 1944.

[26] E.T. Whittaker, G.N. Watson, Modern Analysis, Cambridge University Press, Cambridge, 1935.

[27] P.G. Saffman, G. Baker, Vortex interactions, Ann. Rev. Fluid Mech. 11 (1979) 95

[28] S. Kida, Motion of an elliptic vortex in a uniform shear flow, J. Phys. Soc. Jpn 50 (1981) 3517.

[29] D.W. Moore, P.G. Saffman, Axial flow in laminar trailing vortices, Proc. R. Soc. London, Ser. A 333 (1973) 491.

[30] A.G. Robinson, P.G. Saffman, Three-dimensional stability of vortex arrays, Stud. Appl. Math. 70 (1984) 163.

[31] J.D. Buntine, D.I. Pullin, Merger and cancellation of strained vortices, J. Fluid Mech. 205 (1989) 263.

[32] I. Mezić, A. Leonard, S. Wiggins, Regular and chaotic particle motion near a helical vortex filament, Physica D 111 (1997) 179.

[33] P. Constantin, Geometric statistics in turbulence, SIAM Rev. 36 (1994) 73.

[34] P. Constantin, Ch. Fefferman, A. Majda, Geometric constraints on potentially singular solutions for the 3D Euler equations, Comm. PDE 21 (1996) 559. 